\title{
Copy Number Alterations in Papillary Thyroid Carcinomas: Does Loss of SESN2 Have a Role in Age-related Different Prognoses?
}

\author{
DEISE CIBELE N. DE ALMEIDA ${ }^{1 *}$, MICHEL PLATINI CALDAS DE SOUZA ${ }^{2 *}$, \\ CAROLINA KOURY NASSAR AMORIM ${ }^{3}$, JERSEY HEITOR DA SILVA MAUÉS ${ }^{4}$, FERNANDA DO E. SANTO SAGICA ${ }^{3}$, \\ CAROLINE AQUINO MOREIRA-NUNES ${ }^{5}$ and EDIVALDO HERCULANO C. DE OLIVEIRA ${ }^{3,6}$ \\ ${ }^{1}$ Neuroscience and Cell Biology Pos-Graduation Program, ICB, UFPA, Belém, PA, Brazil; \\ ${ }^{2}$ Center of Technological Innovation, Evandro Chagas Institute, Ananindeua, PA, Brazil; \\ ${ }^{3}$ Laboratory of Tissue Culture and Cytogenetics, SAMAM, Instituto Evandro Chagas, Ananindeua, PA, Brazil; \\ ${ }^{4}$ Laboratory of Human Cytogenetics, Institute of Biological Sciences, Federal University of Pará, Belém, PA, Brazil; \\ ${ }^{5}$ Pharmacogenetics Laboratory, Drug Research and Development Center (NPDM), \\ Federal University of Ceará, Fortaleza, CE, Brazil; \\ ${ }^{6}$ Faculty of Natural Sciences, ICEN, UFPA, Belém, PA, Brazil
}

\begin{abstract}
Background/Aim: Thyroid cancer is the only tumor in which age is an important prognostic factor. In papillary thyroid carcinomas (PTC), 45 years of age seems to be a key point that divides adult patients into two groups, with different clinical features. The aim of the study was to perform a microarray-based analysis in two groups of patients ( $<45$ and $\geq 45$ years old), in order to verify the occurrence of specific copy number alterations (CNAs) that could be associated to different patient behaviors associated with age. Patients and Methods: In order to search and compare genomic alterations that may be related to age, we evaluated the occurrence of CNAs in the genome of 24 PTC samples, divided in two groups $(<45$ and $\geq 45$ years old $)$. Results: We identified only one region showing a statistically significant difference between the groups $(p=0.00357)$ : $a$ deletion of approximately $537 \mathrm{kps}$ in 1p35.3., which was more frequent in patients aged 45 years or older. This is the region where, among others, the gene SESN2 is located,
\end{abstract}

This article is freely accessible online.

*These Authors contributed equally to the study.

Correspondence to: Caroline Aquino Moreira-Nunes, Laboratory of Pharmacogenetics, Drug Research and Development Center (NPDM), Federal University of Ceará, Coronel Nunes de Melo st, n 1000, Rodolfo Teófilo, CEP: 60416-000 Fortaleza, CE, Brazil. Tel: +55 85333668033, e-mail: carolfam@gmail.com

Key Words: Papillary thyroid cancer, aCGH, deletion, SESN2, senescence. which is activated under oxidative stress and plays an antioxidant role, in addition to protecting the genetic material from damage generated by reactive oxygen species (ROS). Conclusion: This is the first time that a CNA involving the deletion of the SESN2 gene is associated with papillary thyroid carcinomas, particularly in patients aged 45 years and older, indicating that this deletion would lead to a more malignant and prominent tumoral behavior associated to a worst prognosis.

Thyroid cancer (TC) is the most common malignancy of the endocrine system, corresponding to $3.4 \%$ of all cancers diagnosed annually worldwide. TC is a unique human tumor, with peculiar biological behavior. This is the only tumor for which age is an important prognostic factor (1). Additionally, $\mathrm{TC}$ affects more frequently women than men. There are five main histological types of TCs: papillary, follicular (which are considered differentiated), poorly differentiated, anaplastic, and medullary TC $(2,3)$.

Of these, papillary thyroid cancer (PTC) is the most frequent among the thyroid neoplasias, representing around $80-85 \%$ of cancers affecting this gland $(4,5)$. PTCs are characterized by presenting a good prognosis, with survival rates of $90 \%$ for 10 years, and show several subtypes, of which the classical variant is the most frequent (6). Among the prognostic factors for PTC, the age of patients is a crucial criterion, because individuals between 20-44 years old present tumors with different behavior when compared to the ones observed in patients older than 45 years (7). Generally, the disease is more aggressive in the second group, with a gradual increase of mortality rates and 
possibility of extrathyroidian extensions, metastases and lymph node involvement (8).

Implementation of customized high-throughput microarraybased comparative genomic hybridization $(\mathrm{aCGH})$ represents an important strategy in the identification of cancer-causing genes and deregulated molecular pathways and hence the identification of biomarkers for different conditions. Copy number alterations (CNAs) have been reported with a low frequency in PTC (9). In addition to point mutations and gene fusions, a significant minority (27.2\%) of PTCs also contained CNAs, which were preferentially present in cases without the most common driver mutations, suggesting a role for CNAs as driver events in this type of tumor (10).

Despite this, the different clinical features related to the age of patients have not been explored in the light of cytogenomic studies. Hence, the aim of this study was to perform a microarray-based analysis in two groups of patients ( $<45$ and $\geq 45$ years old), in order to verify the occurrence of specific CNAs that could be associated to the age range of the patients, and hence justify the different behaviors associated to age.

\section{Patients and Methods}

Patients. Samples of 24 papillary thyroid carcinomas were collected from patients from Belém (Northern Brazil). Of these, 3 were males and 21 females. Concerning age, 12 were $<45$ years old $(2$ males and 10 females) and 12 were $\geq 45$ years old ( 1 male and 11 females). All samples were classified as classical variant subtype, according to histopathological criteria (Table I).

Ethics statement. This study was approved by the Ethics Committee of IEC/SVS/MS (ID 2.611.436), and all patients signed a declaration of consent to permit the use of tumour samples.

Microarray-based comparative genomic hybridization (aCGH). Genomic DNA (gDNA) was isolated using Illustra, Tissue \& cells genomic Prep Mini Spin kit GE Healthcare (Chicago, IL, USA), and aCGH experiments used oligonucleotide-based SurePrint G3 CGH+SNP Array 180K platform (Agilent Technologies, CA, USA), according to manufacturer's instructions. Briefly, $1 \mu \mathrm{g}$ of reference DNA (Agilent Euro Male/Female) and patient DNA were digested and labeled using the SureTag DNA Labeling kit (Agilent Technologies). After purification, labeled sample and reference DNA were co-hybridized at $65^{\circ} \mathrm{C}$ for $16 \mathrm{~h}$ to the array, and microarrays were scanned with Agilent SureScan C Scanner. Features were normalized, extracted using Feature Extraction software (v11.1).

Protein-protein interaction network and enrichment analysis. The SESN2 gene was submitted to a network recovery analysis in the public STRING protein database (v11.0) (http://string-db.org/). A confidence score of 0.40 and a maximum amount of 20 interactions were used. We generated a main protein-protein interaction (PPI) network that was used to identify clusters of interaction modules with the molecular complex detection (MCODE) tool (11). In this step, the following parameters were used in the MCODE (scores $>50$ and the number of nodes $>80$ were set as cutoff criteria with the default
Table I. General information of samples used in the study.

\begin{tabular}{lccc}
\hline ID & Gender & Age & Histopathological classification \\
\hline 1 & F & 30 & PTC \\
2 & F & 78 & PTC \\
3 & F & 47 & PTC \\
4 & F & 72 & PTC \\
5 & F & 28 & PTC \\
6 & F & 46 & PTC \\
7 & M & 51 & PTC \\
8 & F & 33 & PTC \\
9 & F & 29 & PTC \\
10 & F & 51 & PTC \\
11 & F & 61 & PTC \\
12 & F & 32 & PTC \\
13 & F & 17 & PTC \\
14 & F & 47 & PTC \\
15 & F & 72 & PTC \\
16 & F & 40 & PTC \\
17 & F & 35 & PTC \\
18 & F & 49 & PTC \\
19 & M & 36 & PTC \\
20 & F & 45 & PTC \\
21 & F & 38 & PTC \\
22 & F & 20 & PTC \\
23 & F & 64 & PTC \\
24 & M & 41 & PTC \\
\hline
\end{tabular}

F: Female, M: male, PTC: papillary thyroid cancer.

parameters (Degree cutoff $\geq 2$, Node score cutoff $\geq 2$, K-core $\geq 2$ and Max depth=100). This network was validated in the STRING database with species limited to "Homo sapiens" and confidence scores limited to those $>0.4$, to extract PPI data. We used stringApp (http://apps.cytoscape.org/apps/stringapp) to get the functional enrichment of the main module of the PPI network obtained with MCODE. We use a redundancy cutoff $>0.5$ for Gene Ontology (GO) and Kyoto Encyclopedia of Genes and Genomes (KEGG) enrichment. Cytoscape, version 3.8 was used in all analysis (12).

Data analysis and statistics. Data were analyzed and visualized by Agilent Cytogenomics software v2.7 (Santa Clara, CA, USA). Aberration statistical algorithm ADM-2, with threshold 6.0, was used for CNAs. Five-probe 0.15_log2 filter was used for aberration evaluation. Data were manually curated, and the UCSC Genome Bioinformatics was used to eliminate common $\mathrm{CNV}$ regions from the dataset. To compare the frequencies, the Binomial Test for two proportions and the Chi-square of adherence were used. Considering the age groups, the Trend Test was used to verify differences between the proportions of the investigated mutation. Microsoft Excel (Albuquerque, NM, USA), Bioestat 5.3 (Amazonas, Brazil) and PAST 3 (University of Oslo, Oslo, Norway) were used, considering an alpha level of significance of 0.05 .

\section{Results}

The occurrence of papillary thyroid cancer in females was more frequent than males (21/16 and $3 / 24$, respectively), as 
Table II. Distribution of the occurrence of deletion of $1 p 35.3$ in the samples.

\begin{tabular}{lrrr}
\hline Age & \multicolumn{2}{c}{ Deletion } & Total \\
\cline { 2 - 3 } & Yes & No & \\
\hline$<45$ & 4 & 8 & 12 \\
$\geq 45$ & 8 & 4 & 12 \\
Total & 12 & 12 & 24 \\
\hline
\end{tabular}

Results of Chi-square test, with $p<0.5$, comparing the distribution of the occurrence of the deletion of 1 p 35.3 according to the age of the patients.

Table III. Results of binominal test $(Z=0,6122 ; p<0.05)$ and Chi-square test for adherence $(12,042 ; p<0.05)$ comparing the distribution of the occurrence of the deletion of $1 p 35.3$ according to the sex of the patients.

\begin{tabular}{lrrr}
\hline & \multicolumn{2}{c}{ Deletion } & Total \\
\cline { 2 - 3 } & Yes & No & \\
\hline Males & 2 & 1 & 3 \\
Females & 10 & 11 & 21 \\
Total & 12 & 12 & 24 \\
\hline
\end{tabular}

expected. Genome-wide screening showed large genomic heterogeneity in papillary thyroid cancer and revealed a total of 619 CNAs, varying from 14 to 335 per sample, with mean value of 85.8, and standard deviation of 97.3.

Statistically significant differences were not found when considering the two age groups. However, linear correlation analyses demonstrated a negative correlation between age and amplifications. Mean values for deletions and gains were 58.1 and 27.7, respectively (range $=3-256$ and range $=8-82$, respectively). Four samples with the highest CNA numbers showed more deletions than gains.

The statistical analyses of frequencies of each CNA between the two groups showed that only one of them had statistically significant difference $(p=0.00357)$ : a deletion of a segment with approximately $537 \mathrm{~KB}$, located in $1 \mathrm{p} 35.3$ (28547478 to 29085067). This segment was deleted in $8 / 12$ patients with age $\geq 45$ years and only in $4 / 12$ in patients with age $<45$ years. Of the genes located in this region, SESN2 is related to tumor development.

Binominal testing showed that there is a difference between the frequency of mutations in the investigated age groups, preferentially affecting the older ones $(0,33$ in $<45$; 0.67 in $\geq 45 ; Z=1.63 ; p<0.05)$. Trend Chi-square results also proved that there is a difference between the frequencies of deletion in the different investigated age groups (Table II), with a tendency to increase the number of people affected as age advances, under a value of $1.9898 ; p<0.05$ (Figure 1).

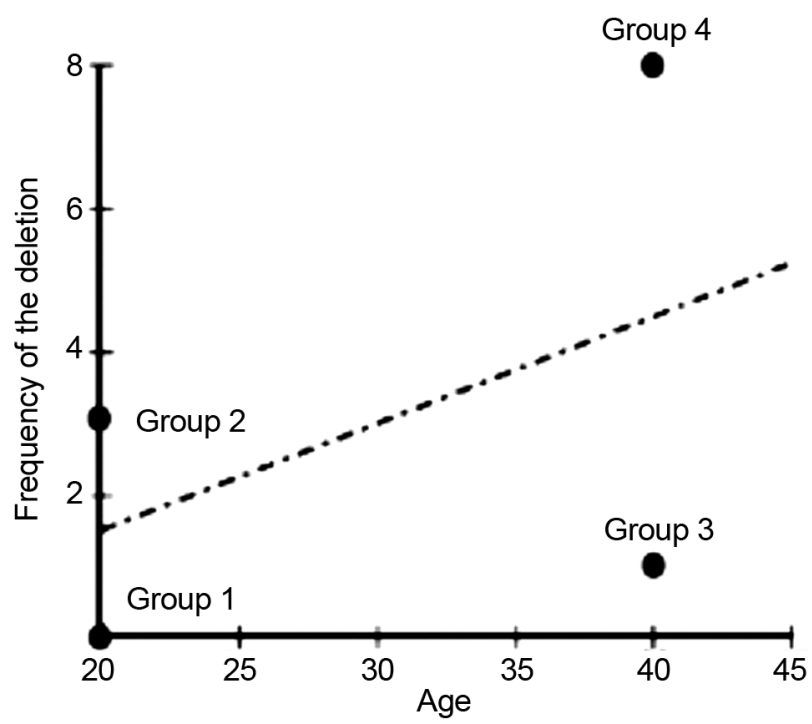

Figure 1. Chi-square results showing a tendency of increase in the number of people affected as age advances, with a value of 1,9898; $p<0,05$.

Additionally, results of binominal test $(\mathrm{Z}=0.6122 ; p>0.05)$ and Chi-square test for adherence $(12.042 ; p<0,05)$ showed that there was no statistically significant difference between sexes (Table III).

Protein-protein network interaction network of SESN2. To identify the biological role and pathways involved with SESN2 network, we performed a PPI network analysis with molecular complex detection revealing a single cluster (Figure 2). This cluster is a network with a topology that contains 19 nodes and 93 edges, which scored a score of 10.33. (Figure 2A). Characteristically, this cluster represents an assortment network, exhibiting the SESN2 as a hub that has connections with other genes strongly connected with an enrichment of $1.0 \mathrm{E}-16$, indicating that the proteins are biologically linked as a group and were noted from: MIOS, MTOR, NPRL2, NPRL3, PRKAA1, PRKAA2, PRKAB1, PRKAB2, PRKAG1, PRKAG2, PRKAG3, SEC13, SEH1L, SESN2, SESN3, SZT2, TP53, WDR24 and WDR59.

The PPI network of this cluster was submitted and validated in the STRING database (Figure 2B). In addition, GO enrichment analysis showed that these genes strongly associated in the biological process category, mainly for (GO: 0032007) regulation of TOR signaling, (GO: 0009267) Cellular response to starvation. In the cell component category for (GO: 0031588) Nucleotide-activated protein kinase complex and (GO: 0035859) Seh1-associated complex and in the molecular function category the highest rank for (GO: 0004679) AMP-activated protein kinase activity (Figure 2C). These genes were also enriched in the KEGG database in a 

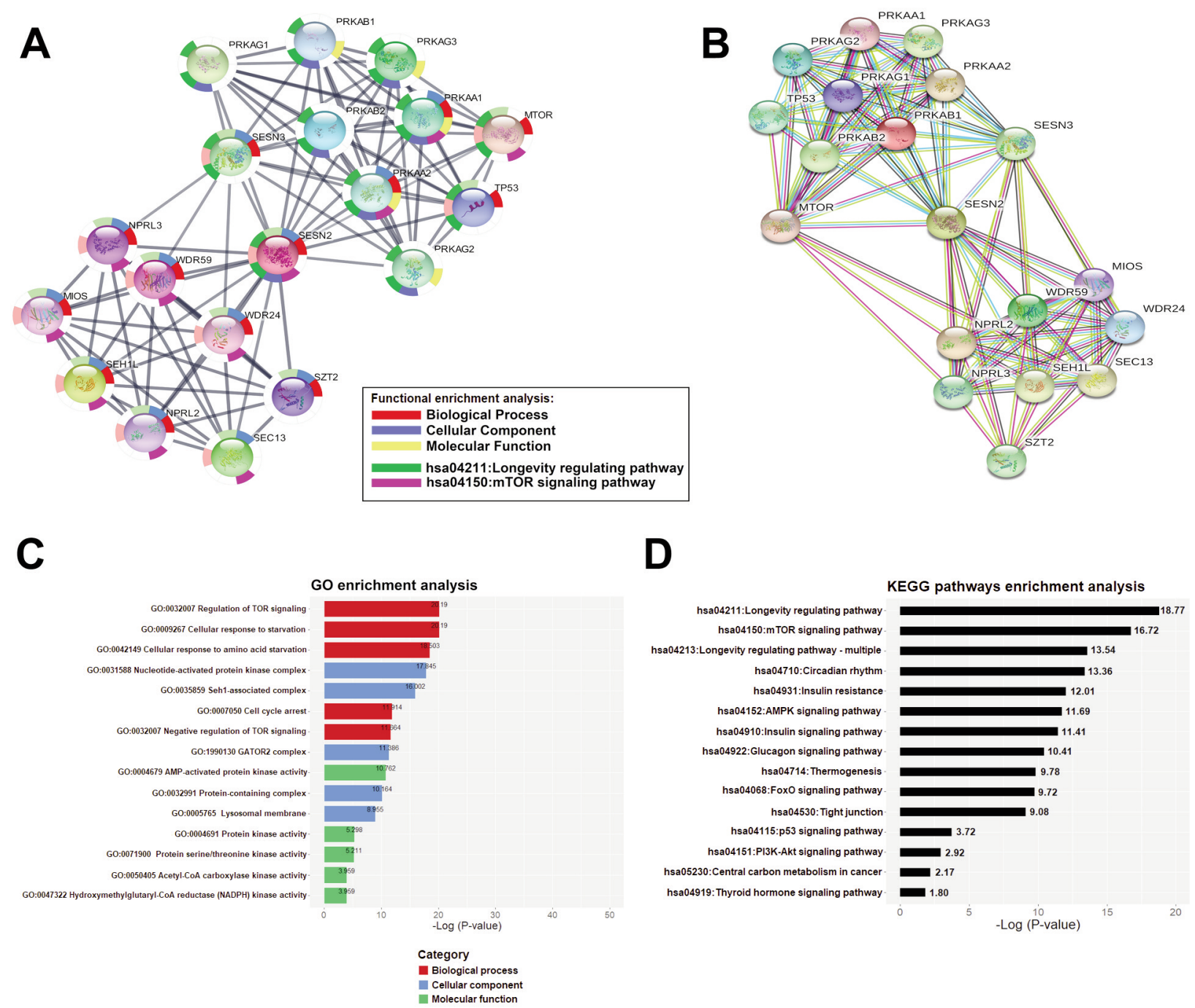

Figure 2. (A) Protein-protein interaction network generated by molecular complex detection from 20 interactions of the SESN2 gene, using Cytoscape software. (B) PPI network validated in the STRING database, confirming two-gene interaction groups separated by the SESN2 hub gene with a PPI enrichment: 1.0E-16 (C) and (D) GO functional annotation for PPI genes showing the most significant functional categories and the most enriched KEGG pathways, respectively. The two groups of gene interactions separated by the SESN2 hub were noted to regulate the two pathways hsa04211: Longevity regulating pathway and hsa04150: mTOR signaling pathway, which have a greater functional enrichment in the KEGG database. All annotation terms were normalized with -Log10 ( $p$-value).

wide repertoire of pathways related to aging processes, signal transduction, growth, metabolism, cell proliferation, autophagy and apoptosis, thyroid cancer, among others (Figure 2D).

\section{Discussion}

SESN2 encodes a protein that is part of a highly conserved family (the so-called Sestrins, composed of the Sesn1, Sesn2 and Sesn3 proteins) (13). When expressed, it negatively regulates the MTOR pathway, which is responsible for the increase of synthesis of proteins and lipids (13-15).
In fact, the PPI network generated in this study showed that SESN2 appears to directly affect the function of a variety of genes, mainly including the regulation of MTOR signaling and also TP53. The activity of MTOR may favor tumor development, since malignant cells require an increment in energy production and acceleration of anabolic processes that support proliferation (15). Thus, SESN2 assists the tumor suppressor function of $\mathrm{p} 53$, by decreasing cellular anabolic activities $(13,14)$. In addition to generate a decrease in energy for the TORC1 synthesis processes, the activation of SESN2 by DNA damage would also divert it to repair it (16). 
SESN2 also plays an antioxidant role and can be activated in response to oxidative stress, regulating the activity of peroxiredoxins, which clean up reactive oxygen species (ROS) or, as already mentioned, inhibiting the TORC1 pathway (16-18). This is an important characteristic to analyze in the context of this study, since ROS rates tend to increase with age.

In certain types of cancers, SESN2 has shown an important role in being pointed out as a tumor suppressor in lung tumors (19), and the decrease in SESN2 expression has also been correlated with a worse prognosis in liver tumors (20) and the induction of its expression restores apoptotic control in head and neck cell models (21).

In this work we described, for the first time in the literature, the deletion of SESN2 in papillary thyroid carcinomas patients aged $\geq 45$ years. SESN2 (and also SESN1) is also often deleted in a variety of cancers. Lee et al. (17) suggested that this fact may imply loss of sestrins in tumor progression related to agedependent accumulation of DNA damage. Indeed, when the nine hallmarks of aging, were proposed by Lópes-Otín et al., one of them presented mitochondrial dysfunction (22). By aging, cells begin to show not only a decline in mitochondrial activities, but a decrease in the efficiency of the respiratory chain, leading, among other things, to increased electron leakage and reduced ATP generation (23). One of the consequences of incorrect mitochondria functioning is the increased production of $\operatorname{ROS}(18,22)$. This fact can be considered physiological, and recent evidence have shown that it may be part of a cellular response in order to maintain its homeostasis (24).

Loss of SESN2 can directly affect ROS levels within cells and in addition, impair normal cellular responses to the stress condition. In a context where cell homeostasis is already affected by aging itself, the loss of SESN2 could play a crucial role in tumor development. An increase in ROS rates, even within a range considered to be non-toxic, would already be advantageous for providing an adaptive response and proliferative stimuli, above this it could also contribute to the occurrence of damage to genetic material, favoring the appearance and accumulation of new mutations $(18,24)$. This could be a possible explanation to more aggressive PTCs in older patients: loss of SESN2 would permit a higher number of mutations.

$R A S$ is a commonly altered gene in PTCs with mutations that lead to its constitutive activity. This abnormal activation leads, among other things, to the so-called Ras-induced ROS accumulation, which is one of the results of the downregulation of the expression of Sesn 1 and Sesn3, leaving only Sesn2 at normal rates to act in the regulation ROS concentrations. The interesting thing is that, in vitro testing in transgenic mouse showed that the increase in ROS levels, by the forced and isolated activation of Ras, induces cellular senescence $(25,26)$.
Considering that additional deregulation would be required for malignant transformation, we suggest the possibility that mutations in $R A S$ and loss of SESN2, thus indicating that all sestrins may interrupt part of the cellular responses activated by 553 and FOXO (the latter activates Sesn3). Thus, the relationship between mutations affecting SESN2 and aggressive PTCs in older patients could be more intense and interconnected than previous techniques allowed to analyze.

\section{Conclusion}

In conclusion, this is the first time that a CNA involving the deletion of the SESN2 gene is associated with papillary thyroid carcinomas, particularly in patients aged 45 years and older, indicating that this deletion would lead to a more malignant and prominent tumoral behavior associated to a worst prognosis. In addition, the evaluation of pathways related to responses to oxidative stress would also be interesting in this scenario, since, based on the findings described in this work, this seems to be an element of importance for tumor development in aged patients.

\section{Conflicts of Interest}

The Authors declare that they have no conflicts of interest.

\section{Authors' Contributions}

DCNA and MPCS conceived the study and were in charge of overall direction and planning. DCNA contributed to sample collection, preparation and data curation. CKNA performed aCGH experiments, data scanning and organization of information. FESS performed statistical analyses. JHSM and CAMN performed bioinformatics analysis. MPCS, CAMN and EHCO contributed to the interpretation of the results. MPCS, DCNA, CAMN and EHCO wrote the manuscript. All Authors read and approved the final article.

\section{Acknowledgements}

The Authors would like to thank Instituto Evandro Chagas/SVS/MS and PAPQ-PROPESP (UFPA) for technical and financial support, and CAPES/CNPq. We also would like to thank the hospital staff, and patients whose support and collaboration made this study possible. This study was partially supported by the Ministry of Health through the Evandro Chagas Institute official budget and by CNPq (process 307382/2019-2).

\section{References}

1 Shaha AR and Tuttle RM: Thyroid cancer staging and genomics. Ann Transl Med 7: S49, 2019. PMID: 31032328. DOI: 10.21037/atm.2019.03.11

2 Stanta G, Jahn SW, Bonin S and Hoefler G: Tumour heterogeneity: principles and practical consequences. Virchows Arch 469: 371-384, 2016. PMID: 27412632. DOI: 10.1007/ s00428-016-1987-9 
3 Chmielik E, Rusinek D, Oczko-Wojciechowska M, Jarzab M, Krajewska J, Czarniecka A and Jarzab B: Heterogeneity of thyroid cancer. Pathobiology 85: 117-129, 2018. PMID: 29408820. DOI: $10.1159 / 000486422$

4 Cooper DS, Doherty GM, Haugen BR, Kloos RT, Lee SL, Mandel SJ, Mazzaferri EL, Mclver B, Sherman SI and Tuttle R: Management guidelines for patients with thyroid nodules and differentiated thyroid cancer. Thyroid 16(2): 109-141, 2006. PMID: 16420177. DOI: 10.1089/thy.2006.16.109

5 Sipos JA and Mazzaferri EL: Thyroid cancer epidemiology and prognostic Variables. Clin Oncol 22: 395-404, 2010. PMID: 20627675. DOI: 10.1016/j.clon.2010.05.004

6 Grubbs EG, Rich TA, Li G, Sturgis EM, Younes MN, Myers JN, Edeiken- Monroe B, Fornage BD, Monroe DP, Staerkel GA, Williams MD, Waguespack SG, Hu MI, Cote G, Gagel RF, Cohen J, Weber RS, Anaya DA, Holsinger FC, Perrier ND, Clayman GL and Evans DB: Recent advances in thyroid cancer. Curr Probl Surg 45(3): 149-151, 2008. PMID: 18346476. DOI: 10.1067/j.cpsurg.2007.12.008

7 Davies L, Morris LG, Haymart M, Chen AY, Goldenberg D, Morris J, Olgivie JB, Terris DJ, Netterville J, Wong RJ and Randolf G: American association of clinical endocrinologists and american college of endocrinology disease state clinical review: the increasing incidence of thyroid cancer. Endocr Pract 21: 686696, 2015. PMID: 26135963. DOI: 10.4158/EP14466.DSCR

8 Haymart MR: Understanding the relationship between age and thyroid cancer, Oncologist 14(3): 216-221, 2009. PMID: 19270027. DOI: 10.1634/theoncologist.2008-0194

9 Nikiforov YE and Nikiforova MN: Molecular genetics and diagnosis of thyroid cancer. Nat Rev Endocrinol 7(10): 569-580, 2011. PMID: 21878896. DOI: $10.1038 /$ nrendo.2011.142

10 Giordano TJ: Genomic hallmarks of thyroid neoplasia. Annu Rev Pathol 13: 141-162, 2018. PMID: 29083981. DOI: 10.1146/annurev-pathol-121808-102139

11 Bader GD and Hogue CWV: An automated method for finding molecular complexes in large protein interaction networks. BMC Bioinformatics 4(2): 81-90, 2003. PMID: 12525261. DOI: 10.1186/1471-2105-4-2

12 Shannon P, Markiel A, Ozier O, Baliga NS, Wang JT, Ramage D, Amin N, Schwikowski B and Ideker T: Cytoscape: A software environment for integrated models of biomolecular interaction networks. Genome Res 13(11): 2498-2504, 2003. PMID: 14597658. DOI: $10.1101 /$ gr.1239303

13 Luo C, Zhao S, Zhang M, Gao Y, Wang J, Hanigan MD and Zheng N: SESN2 negatively regulates cell proliferation and casein synthesis by inhibition the amino acid-mediated mTORC1 pathway in cow mammary epithelial cells. Sci Rep 8: 3912, 2018. PMID: 29500367. DOI: 10.1038/s41598-018-22208-w

14 Jeong S, Kim D, Kang SH, Yun HK, Kim JL, Kim BR, Park SH, $\mathrm{Na}$ YJ, Jo MJ, Jeong YA, Kim BG, Lee DH and Oh SC: Docosahexaenoic acid enhances oxaliplatin-induced autophagic cell death via the ER Stress/Sesn2 pathway in colorectal cancer. Cancers 11: 982, 2019. PMID: 31337142. DOI: 10.3390/cancers 11070982

15 Gonzalez S and Rallis C: The TOR signaling pathway in spatial and temporal control of cell size and growth. Front Cell Dev Biol 5: 61, 2017. PMID: 28638821. DOI: 10.3389/fcell.2017.00061
16 Parmigiani A, Nourbakhsh A, Ding B, Wang W, Kim YC, Akopiants K, Guan KL, Karin M and Budanov AV: Sestrins inhibit mTORC1 kinase activation through the GATOR complex. Cell Rep 9: 1281-1291, 2014. PMID: 25457612. DOI: 10.1016/j.celrep.2014.10.019

17 Lee JH, Bodmer R, Bier E and Karin M: Sestrins at the crossroad between stress and aging. Aging 2(6): 369-374, 2010. PMID: 20606249. DOI: 10.18632/aging.100157

18 Quan N, Sun W, Wang L, Chen X, Bogan JS, Zhou X, Cayes C, Liu Q, Zheng Y and Li J: Sestrin2 prevents age-related intolerance to ischemia and reperfusion injury by modulating substrate metabolism. FASEB J 31: 4153-4167, 2017. PMID: 28592638. DOI: $10.1096 / f j .201700063 R$

19 Xu H, Sun H, Zhang H, Liu J, Fan F, Li Y, Ning X, Sun Y, Dai S, Liu B, Gao M, Fu S and Zhou C: An shRNA based genetic screen identified Sesn2 as a potential tumor suppressor in lung cancer via suppression of Akt-mTOR-p70S6K signaling. PloS One 10(5): 117, 2015. PMID: 25962159. DOI: 10.1371/journal.pone.0124033

20 Chen S, Yan W, Lang W, Yu J, Xu L and Xu X: SESN2 correlates with advantageous prognosis in hepatocellular carcinoma. Diagn Pathol 12(1): 13, 2017. PMID: 28118855. DOI: $10.1186 / \mathrm{s} 13000-016-0591-2$

Won DH, Chung SH, Shin JA, Hong KO, Yang IH, Yu JW and Cho SD: Induction of sestrin 2 is associated with fisetin-mediated apoptosis in human head and neck cancer cell lines. J Clin Biochem Nutrit 64(2): 97-105, 2019. PMID: 30936621. DOI: $10.3164 /$ jcbn.18-63

22 López-Otín C, Blasco MA, Partridge L, Serrano M and Kroemer G: The hallmarks of aging. Cell 153, 2013. PMID: 23746838. DOI: 10.1016/j.cell.2013.05.039

23 Green DR, Galluzzi L and Kroemer G: Mitochondria and the autophagy-inflammation-cell death axis in organismal aging. Science 333: 1109-1112, 2011. PMID: 21868666. DOI: $10.1126 /$ science 1201940

24 Sena LA and Chandel NS: Physiological roles of mitochondrial reactive oxygen species. Mol Cell 48(2): 158-167, 2012. PMID: 23102266. DOI: 10.1016/j.molcel.2012.09.025

25 Kopnin PB, Agapova LS, Kopnin BP and Chumakov PM: Repression of sestrin family genes contributes to oncogenic Rasinduced reactive oxygen species up-regulation and genetic instability. Cancer Res 67: 4671-4678, 2007. PMID: 17510393. DOI: $10.1158 / 0008-5472 . C A N-06-2466$

26 Serrano M, Lin AW, McCurrach ME, Beach D and Lowe SW: Oncogenic Ras provokes premature cell senescence associated with accumulation of p53 and p16INK4a. Cell 88: 593-602, 1997. PMID: 9054499. DOI: 10.1016/s0092-8674(00)81902-9

Received June 14, 2020

Revised July 1, 2020

Accepted July 7, 2020 